\title{
Diseño de un sistema fotovoltaico para la reducción de usuarios de tarifa DAC
}

\section{Design of a photovoltaic system for reducing "DAC" rate users}

\author{
SÁNCHEZ-CORTEZ, José Alfonso †*, CASTILLO-RAMÍREZ, Carlos Eduardo, VÁZQUEZ- \\ BALDAZO, Luis Guillermo y GONZALEZ-MORALES, Amparo
}

Universidad Tecnológica de Altamira

ID $1^{\mathrm{er}}$ Autor: José Alfonso, Sánchez-Cortez / ORC ID: 0000-0002-8762-1154, CVU CONACYT ID: 500152

ID $1^{\mathrm{er}}$ Coautor: Carlos Eduardo, Castillo-Ramírez / ORC ID: 0000-0003-2378-4226, CVU CONACYT ID: 1001435

ID $2^{\text {do }}$ Coautor: Amparo, González-Morales / ORC ID: 0000-0003-2378-4226, CVU CONACYT ID: 1001435

ID $3^{\text {er }}$ Coautor: Luis Guillermo, Vázquez-Baldazo / ORC ID: 0000-0001-5616-5339, CVU CONACYT ID: 1019622

DOI: $10.35429 /$ JSI.2019.11.3.1.9

Recibido 02 de Junio, 2019; Aceptado 30 de Septiembre, 2019

\section{Resumen}

El incremento en los costos por consumo de energía eléctrica ha ido al alza, afectando considerablemente la economía de los usuarios, dentro de las tarifas de usuarios residenciales se encuentra la tarifa DAC (Servicio Doméstico de Alto Consumo). Un análisis económico en las tarifas de consumo de electricidad indica que, a partir del 1 de enero de 2019, el costo por cada $\mathrm{kWh}$ que se utiliza en tarifa DAC corresponde a $\$ 5.121$ esto significa que un usuario DAC paga más del $200 \%$ que un usuario $1 \mathrm{C}$ por cada $\mathrm{kWh}$ consumido. Para ello se ha diseñado un sistema fotovoltaico para interconexión a la red, este sistema permite a los usuarios cambiar de tarifa DAC a tarifa $1 \mathrm{C}$ y en consecuencia permitirá reducir sus costos por el servicio de energía eléctrica, dicho sistema puede ser instalado en cualquier jardín, y además de generar energía eléctrica, los productos fotovoltaicos serán 2: un columpio y un gazebo, con una capacidad instalada de $1.1 \mathrm{kWh}$, mismos que proveerá de un servicio de descanso y relajación.

Tarifa DAC, Tarifa 1C, kWh

\begin{abstract}
The increase in costs for electricity consumption has gone up, significantly affecting the economy of users, within the tariffs of residential users is the "DAC" rate (that means high consumption domestic rate). An economic analysis of electricity consumption rates indicates that, as of January 1st, 2019, the cost per kWh used in the "DAC" tariff corresponds to $\$ 5.121 \mathrm{MXN}$, which means that a "DAC" user pays more than $200 \%$ than a user $1 \mathrm{C}$ rate for each $\mathrm{kWh}$ consumed. For this purpose, a photovoltaic system has been designed for interconnection to the network, this system allows users to change from "DAC" rate to tariff $1 \mathrm{C}$, and consequently will allow the reduction of these costs about the electric power service, this system can be installed in any yard, besides generating electricity, the photovoltaic products will be 2 : a swing and a gazebo, with an installed capacity of $1.1 \mathrm{kWh}$, which will provide a rest and relaxation service.
\end{abstract}

Tarifa DAC, Tarifa 1C, kWh

Citación: SÁNCHEZ-CORTEZ, José Alfonso, CASTILLO-RAMÍREZ, Carlos Eduardo, VÁZQUEZ-BALDAZO, Luis Guillermo y GONZALEZ-MORALES, Amparo. Diseño de un sistema fotovoltaico para la reducción de usuarios de tarifa DAC. Revista de Innovación Sistemática. 2019. 3-11:1-9

\footnotetext{
*Correspondencia al Autor (Correo electrónico: jsanchez@utaltamira.edu.mx)

$\dagger$ Investigador contribuyendo como primer Autor.
} 


\section{Introducción}

En 2017 el consumo de energía en el país se incrementó un $31.6 \%$ respecto al año anterior, y el consumo per cápita en este mismo año se incremento un $0.2 \%$ respeto al 2016 , es decir que se registró un consumo de 74.89 GJ, el incremento de la población fue el principal factor del incremento de energía, ya que la población mexicana creció un $1.0 \%$ entre el 2016 y 2017 pasando de 112.27 a 123.52 millones. (SENER, Balance Nacional de Energia, 2018) La siguiente grafica demuestra lo mencionado.

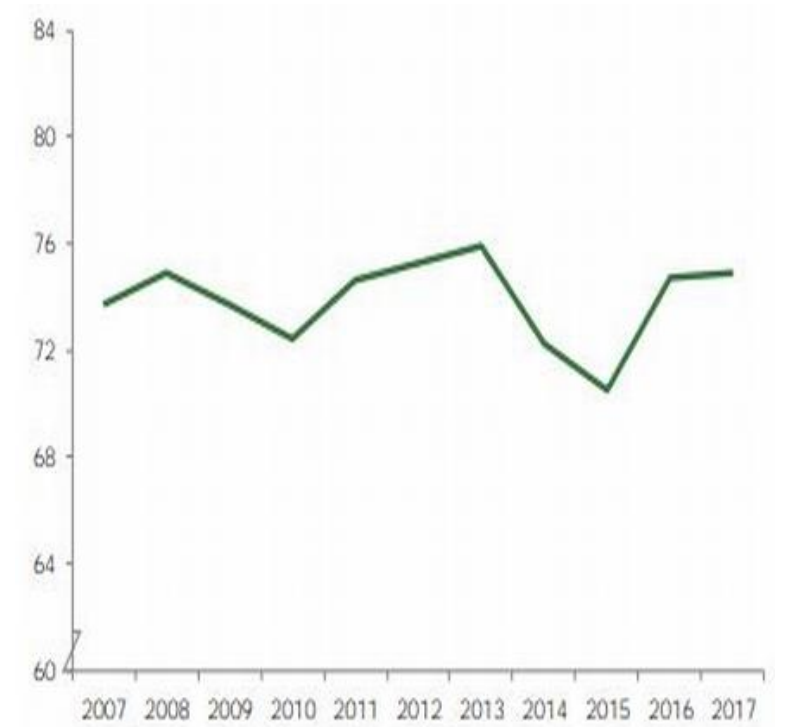

Gráfico 1 Consumo de energía "per cápita" (en GJ) por habitante

De acuerdo con el Balance Nacional de Energía 2017 “El petróleo crudo es el energético que ocupó la fracción más grande de la producción nacional, con 4,354.89 PJ". Esto representa el $62.0 \%$ de producción de energía mediante distintos recursos. En cambio, las energías renovables representan un porcentaje más bajo, el 3.9\%. (SENER, Balance Nacional de Energía 2017, 2018) México tiene un gran potencial para el desarrollo de centrales fotovoltaicas y generación distribuida, en el 2017 se registró un incremento del 36.7\% respecto al 2016. (SENER, Balance Nacional de Energía, 2018).

\section{Consumo de energía a nivel nacional.}

El consumo energético a nivel nacional no es proporcionado, por ende, las tarifas de consumo tienen la misma afectación, dentro otros factores el más importante son las temperaturas, en zonas cálidas se incrementa la tarifa y el consumo respecto a zonas templadas.
Un informe publicado en 2018 cita lo siguiente: "El consumo en la primera zona llega a ser 2 o 3 veces mayor comparado en zonas templadas. Un rasgo muy importante que evaluar es el equipamiento de sistemas que necesitan electricidad, dado que se utilizan diferentes equipos para cada zona según la necesidad.

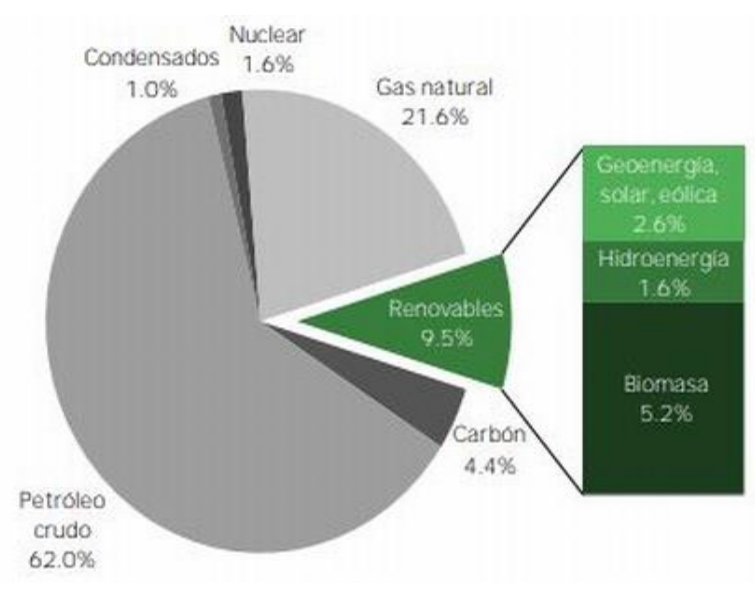

Gráfico 2 Porcentaje de fuentes de energía en México

\section{Tarifa DAC}

Comisión Federal de Electricidad define lo siguiente: "Esta tarifa se aplicará a los servicios que destinen la energía para uso exclusivamente doméstico, individualmente a cada residencia, apartamento, apartamento en condominio o vivienda, considerada de alto consumo o que por las características del servicio así se requiera." (Comisión Federal de Electricidad, 29) Cuando un usuario "entra" en tarifa DAC es porque ha rebasado el consumo mensual (en $\mathrm{kWh}$ ) establecido de acuerdo con su tarifa y región.

El aumento de usuarios que se encuentran en tarifa DAC es alarmante, son diversos los factores:

- Uso de electrodomésticos antiguos y/u obsoletos, que consumen mucha más energía que los actuales.

Fugas de energía causado de una instalación eléctrica mala o deficiente. Mala educación energética.

- Uso excesivo de equipos de aire acondicionado. 
En zonas cálidas del país es más frecuente el incremento de usuarios en DAC, debido a las altas temperaturas del verano, en los meses más calurosas de esta temporada se han registrado hasta $40^{\circ} \mathrm{C}$ a la sombra, por ende, los usuarios que disponen de aire acondicionado hacen uso de este y (en ocasiones hacen uso excesivo de este aparato), en este último caso, el consumo normal sumado a esta carga excesiva (o de más) provoca un incremento considerable en el consumo de energía y su repercusión es directa en la factura de energía.

\section{Tarifas de CFE}

A partir del 1o de enero de 2019, el costo por cada kWh que se utiliza en casa corresponde: a $\$ 5.121$ (respectivo a que la tarifa de la zona en DAC) tabla 1; es decir $25 \%$ más respecto a hace un año, y $10 \%$ respecto al mes anterior. Retomando el costo del kWh en el mes de enero en la tarifa DAC (\$5.121) comparando este con el costo de la energía en la tarifa 1C:

- $\quad$ (\$ 0.796 por cada uno de los primeros 75 $\mathrm{kWh}$

$\$ 0.960$ por cada uno de los siguientes $100 \mathrm{kWh} \$ 2.813$ por cada kilowatt-hora adicional a los anteriores)

Se puede observar que la tarifa DAC es por lo menos $54.93 \%$ más cara que la tarifa $1 \mathrm{C}$. (Cortez, 2019)

\begin{tabular}{|c|c|c|}
\hline Región & $\begin{array}{l}\text { Cargo } \\
\text { fijo }\end{array}$ & $\begin{array}{l}\text { Cargo por energía } \\
\text { consumida }(\$ / \mathrm{kWh})\end{array}$ \\
\hline Central & $\$ 107.58$ & $\$ 5.469$ \\
\hline Noro Este & $\$ 107.58$ & $\$ 5.121$ \\
\hline Norte y Noreste & $\$ 107.58$ & $\$ 4.993$ \\
\hline Sur y Peninsular & $\$ 107.58$ & $\$ 5.070$ \\
\hline
\end{tabular}

Tabla 1 Tarifas de CFE según la región

\section{Costo de tarifas a nivel nacional.}

El factor principal causante del a tarifa DAC es que los usuarios sobrepasan el consumo (en $\mathrm{kW} / \mathrm{h}$ ) establecido por CFE. Cada usuario recibe una cantidad de $\mathrm{kW}$ de acuerdo con su tarifa y el precio varía acorde a la región del país.

Debido a que es muy elevado el costo de esta tarifa (ya que los usuarios que se encuentran dentro de ella pagan hasta un $200 \%$ más de las tarifas habituales) que afecta severamente su economía.
De entre las múltiples opciones para erradicar esta situación, una de ellas es:

- $\quad$ Hacer eficientes al máximo los aparatos consumidores actuales del hogar. Esto significa eliminar aparatos viejos por modernos que ahorran energía, y mantener durante un largo tiempo un consumo bajo para reducir el promedio anual que le daría entrada a la tarifa subsidiada.

- Esto va de la mano con la correcta forma de utilizar la energía eléctrica y evitar desperdiciarla, se refiere a: evitar dejar cargadores conectados, apagar las lámparas que no se estén utilizando, reducir los tiempos de uso del aire acondicionado etc.

\section{Soluciones}

Una de las soluciones más frecuente es la instalación de sistemas fotovoltaicos. De acuerdo con la configuración que más le convenga al usuario, este sistema permite generar su propia energía y la que no es utilizada por el cliente:

Se almacena en un banco de baterías, (que sirve como respaldo energético) o bien, se inyecta a la red de la compañía suministradora de energía (En base al Manual de interconexión de centrales de generación con capacidad menor a $0.5 \mathrm{MW}$, capitulo 2 Generación distribuida, subtema: Actividades de los generadores cito lo siguiente: "Venta de excedentes de la energía eléctrica: Se refiere a la generación de energía eléctrica que excede la satisfacción de las necesidades de los Centros de Carga, y que es inyectada a las Redes Generales de Distribución para ser vendida"). (Comision Reguladora de Energía., 2017)

\section{Objetivos}

- Realizar análisis energéticos y evaluar si realmente el usuario necesita un sistema fotovoltaico o si no, proporcionarle otras opciones.

- Proporcionar a los usuarios con tarifa DAC una solución óptima, factible y adecuada a su necesidad. 


\section{Contribución de la investigación}

Las instalaciones fotovoltaicas comunes normalmente se implementan en techos, azoteas etc. De acuerdo con las necesidades energéticas de los usuarios, será el dimensionamiento del sistema fotovoltaico (sea aislado o interconectado), por ende, requiere un espacio destinado para todo el equipo de la instalación. Si la casa o lugar no dispone de este espacio, será muy complicado realizar la instalación o se tendrá que hacer modificaciones estructurales, lo que conlleva a una inversión extra para el usuario.

En ocasiones por "recomendación" y/o elección propia del cliente, este hace uso de "kits" o "paquetes solares" que le prometen disminuir su consumo de energía y por ende a reducir el pago de su tarifa de energía. Por lo general, cuando esta elección es así, sin antes haber realizado un estudio (y posteriormente un diagnostico) energético, el cliente adquiere este "paquete" para "resolver" el consumo excedente de energía, la mayoría de las veces, estos van dirigidos a los aires acondicionados, el cual es el equipo que más energía consume en un hogar (considerando los factores de uso)

En este caso, el problema es que el cliente va a invertir en un sistema que solo va a "atacar" una parte del problema y no lo va a solucionar por completo. Es por ello, se recomienda realizar los estudios previos a una instalación de este tipo y darle la solución al cliente que realmente necesita.

\section{Concepto del gazebo.}

Se ha idealizado o conceptualizado como producto de penetración en el mercado de consumo, siendo este primero un gazebo (de dimensiones similares a lo que se comercializan) de PTR (perfil tubular rectangular) en cuyo techo, o parte superior estará cubierto con material fotovoltaico, con una capacidad instalada de $1.1 \mathrm{~kW}$

Este dispositivo (ya instalado) permitirá al usuario disfrutar de una zona de descanso y relajación que este le brindará y, por otro lado, el dispositivo estará generando energía eléctrica limpia. (Ruiz, 2018). Se estima una producción de $1.10 \mathrm{~kW}$ que se va a inyectar a la red de CFE Con este producto se pretende atacar tres factores importantes:
- Que el usuario salga de tarifa DAC.

- Proporcionar una zona de confort y descanso mientras genera energía eléctrica limpia.

- Erradicar los espacios inutilizables por los módulos fotovoltaicos convencionales.

\section{Desarrollo del proyecto}

Durante el primer semestre del año 2018 en la zona conurbada de Tamaulipas, que comprende a los municipios de: Tampico, madero y Altamira la cantidad de usuarios dentro de tarifa DAC que se registró por municipio (en promedio) fue la siguiente: (México., 2018)

\begin{tabular}{|l|r|}
\hline Municipio & Promedio de usuarios en DAC \\
\hline Altamira & 540 \\
\hline Tampico & 153 \\
\hline CD Madero & 122 \\
\hline
\end{tabular}

Tabla 2 Cantidad de usuarios en DAC

Siendo el municipio de tampico el que contaba con más usuarios "afectados", teniendo en cuenta esta problemática, se decidió optar por la realización de este proyecto, cuya intención principal es brindar una solución a estos usuarios principalmente y así ayudarlos a salir de esta problemática. A partir de aquí se comienza con la planeación, diseño y elaboración del primer prototipo "teórico" donde estarán participando alumnos de la Universidad Tecnológica de Altamira, de la carrera de Energías Renovables, nivel de Ingeniería. Esto con la finalidad de promover en los alumnos el logro de habilidades medibles a lo largo de sus estudios, que los sumerjan en un clima real de prácticas. Durante el desarrollo de este proyecto, los alumnos fueron obteniendo y puliendo habilidades tales como:

\section{- Conexiones eléctricas.}

- Corte y soldado de piezas metálicas.

- $\quad$ Uso de softwares de diseño en 3D ${ }^{\circledR}$.

- Uso de equipo fotovoltaico (módulos, inversores, aparatos de medición, etc.)

Una de las características de este prototipo es que será "desmontable" (la mayoría de sus partes) facilitando así su trasportación, es preciso decir que solo personal autorizado podrá hacer el desmantelamiento y transportación de los equipos hacia donde el usuario indique, por lo tanto, la seguridad de los usuarios no será comprometida.

SÁNCHEZ-CORTEZ, José Alfonso, CASTILLO-RAMÍREZ, Carlos Eduardo, VÁZQUEZ-BALDAZO, Luis Guillermo y GONZALEZMORALES, Amparo. Diseño de un sistema fotovoltaico para la reducción de usuarios de tarifa DAC. Revista de Innovación Sistemática. 2019 
El desarrollo del artículo se plantea seccionado en 5 partes y son las siguientes:

$\begin{array}{ll}- & \text { Estudio del lugar } \\ - & \text { Ubicación. } \\ - & \text { Memoria de cálculo. } \\ - & \text { Normativa utilizada. } \\ - & \text { Factor económico. (Amortización) }\end{array}$

\section{Estudio del lugar}

Los aspectos importantes para el desarrollo y funcionamiento óptimo del proyecto son los siguientes: cantidad de HPS, clima, localización.

\section{Insolación promedio a $22^{\circ}$}

$4.99 \mathrm{~h}$

\section{Insolación promedio en ángulo óptimo}

\section{$5.17 \mathrm{~h}$}

Figura 1 Horas Pico Solar (Altamira, Tamps.)

Estos datos serán necesarios al momento de realizar los cálculos y el dimensionamiento correspondiente del proyecto, y así obtener el máximo rendimiento de la instalación.

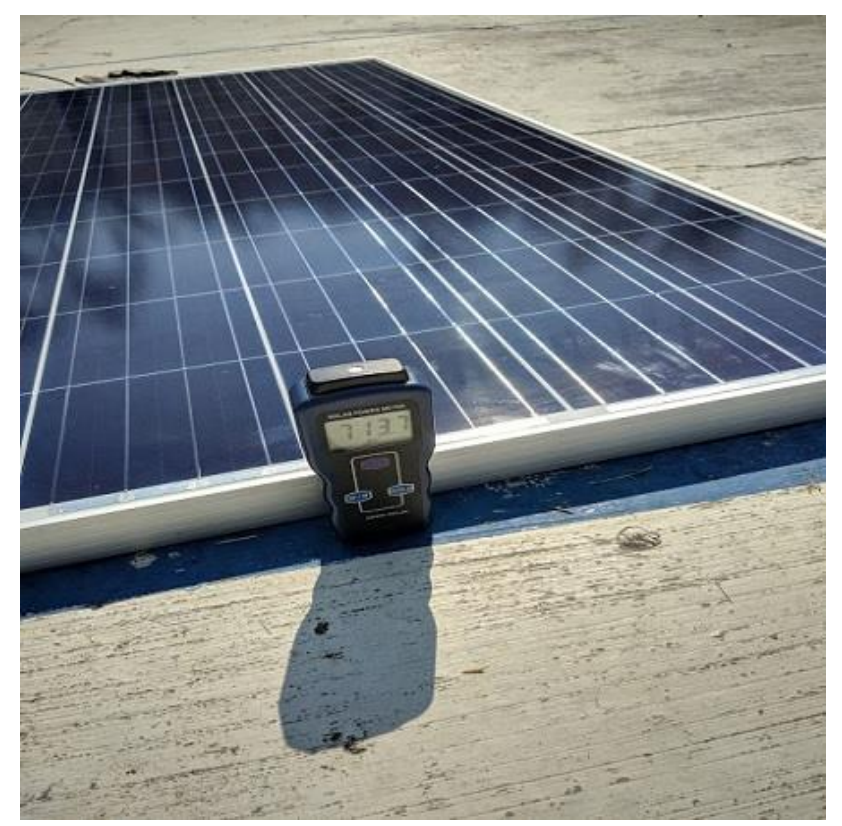

Figura 2 Medido de irradiancia en la zona
Este este caso, el proyecto se llevará a cabo en la ciudad de Altamira Tamaulipas, en donde el recurso solar es abundante, esto no quiere decir que el proyecto solo es exclusivo y/o destinado a Altamira, sino a todo el estado, cuenta con un recurso solar muy valioso, el estado de Tamaulipas recibe en promedio 3500 Wh/m2/día. ((SENER), 2019)

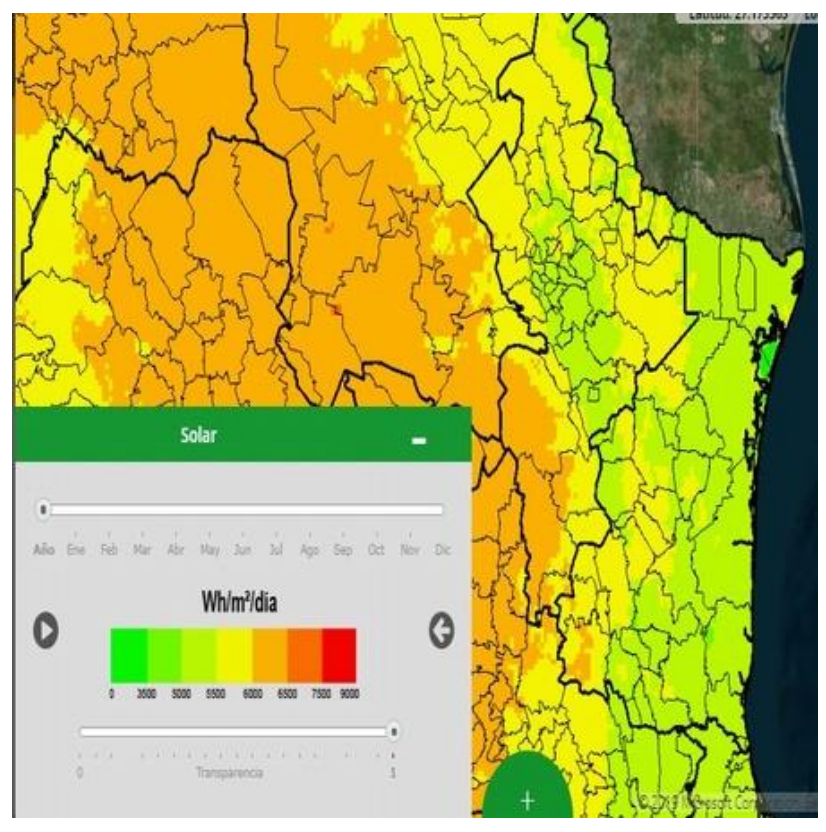

Figura 3 Recurso Solar de Tamaulipas

\section{Ubicación}

Se desarrollará en las instalaciones de la universidad tecnológica de Altamira, en el laboratorio de máquinas eléctricas., siendo aquí donde se desarrollará la mayor parte del proyecto.

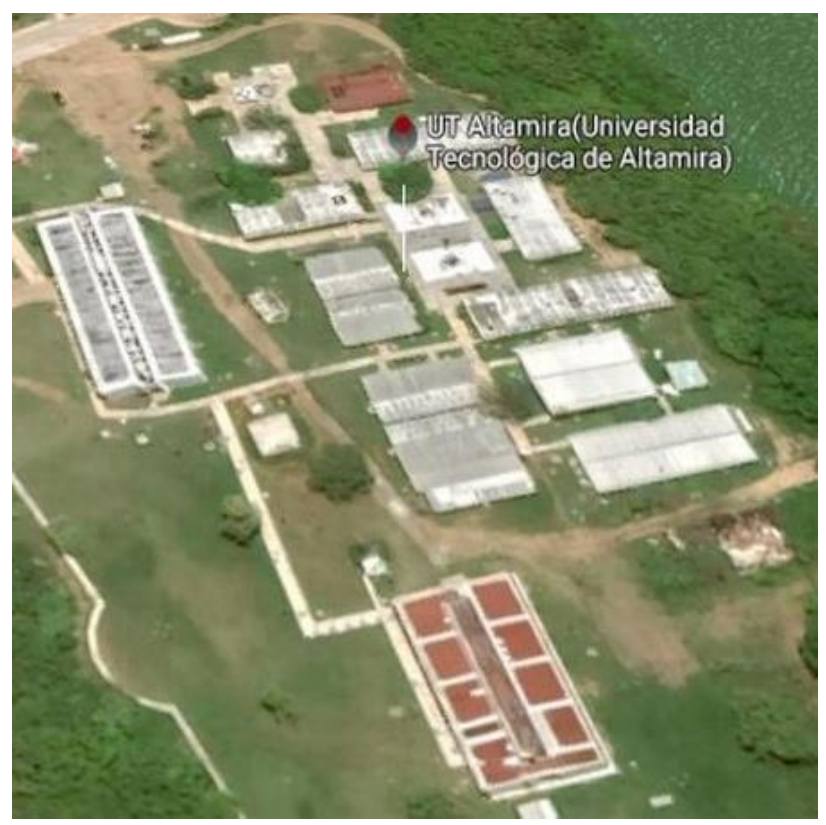

Figura 4 Ubicación y vista aérea de la universidad

SÁNCHEZ-CORTEZ, José Alfonso, CASTILLO-RAMÍREZ, Carlos Eduardo, VÁZQUEZ-BALDAZO, Luis Guillermo y GONZALEZMORALES, Amparo. Diseño de un sistema fotovoltaico para la reducción de usuarios de tarifa DAC. Revista de Innovación Sistemática. 2019 


\section{Memoria de cálculo}

Para diseñar una instalación fotovoltaica acorde a las necesidades del cliente, es preciso realizar cálculos matemáticos con mucha exactitud, ya que esto nos permitirá tener un rendimiento óptimo de la instalación y a la postre evitar pérdidas de energía. Es importante recalcar que, la capacidad mínima de un sistema voltaico interconectad a la red requerida por CFE es de $0.5 \mathrm{KW}$. (eléctrica., 2011)

\section{Datos del módulo}

Para el concepto del gazebo se diseñará una instalación teórica de $1.11 \mathrm{KWh}$, en este caso los módulos se instalarán en la misma estructura del gazebo, por lo cual no será necesario una estructura adicional. Se utilizarán 3 módulos fotovoltaicos de las siguientes características:

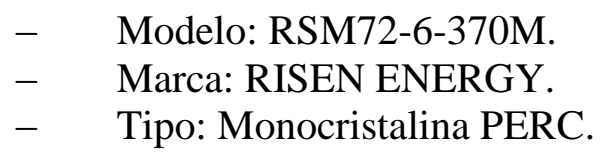
por el FIDE.

Este módulo está aprobado y certificado

\begin{tabular}{|l|r|}
\multicolumn{1}{|c|}{ Datos técnicos } & Valores \\
\hline Potencia STC & 370 \\
\hline Voltaje máximo & 39.8 \\
\hline Corriente máxima & 9.3 \\
\hline Voltaje en circuito abierto & 48.4 \\
\hline Corriente en corto circuito & 9.9 \\
\hline Numero de celdas & 72 \\
\hline Coeficiente de temperatura. & $-0.29 \% /{ }^{\circ} \mathrm{C}$ \\
\hline
\end{tabular}

Tabla 3 Datos del módulo fotovoltaico

\section{Elección del inversor}

Para este caso, se necesitará un inversor de corriente de DC/AC, en donde solo se usará un string o cadena debido tamaño de la instalación, como dato principal; se toma la potencia nominal de cada módulo, la que se multiplica por el número de módulos instalados y un factor de protección, en este caso del 25\% más de potencia. Entonces el cálculo se realiza con la siguiente formula:

$\mathrm{PT}=\mathrm{Wp} * \mathrm{M} * \mathrm{f} . \quad \mathrm{p}$

Donde:

(1) $\mathrm{Wp}=$ Potencia del modulo

(2) $M=$ Numero de módulos.

(3) f.p = Es un factor de protección del 1.25\%
Para este caso, se utiliza como dato la corriente en corto circuito del módulo cuyo valor en este caso es de: 9.9 amperes, otro dato a recordar es la cantidad de strings, en este caso solo es uno.

\section{Normativa}

Para realizar este cálculo se debe tener en cuenta el uso y cumplimiento de la norma oficial mexicana: NOM-001-SEDE-2012, Instalaciones Eléctricas, Articulo 690, sección 8. En esta sección se realizará el cálculo para obtener los conductores adecuados para el sistema, se utilizará conductor (de cobre o aluminio) tipo USE-2 XLPE en cumplimiento con la NOM001-SEDE-2012(instalaciones eléctricas). Para esto se realizará el cálculo y se muestra a continuación.

\begin{tabular}{|l|r|}
\hline \multicolumn{1}{|c|}{ Datos } & \multicolumn{1}{c|}{ Valores } \\
\hline Potencia total & 1387.5 \\
\hline Voltaje de operación & 110 \\
\hline Amperes del sistema & 12.6136364 \\
\hline Caída de tensión (5\%) & 5.5 \\
\hline Resistividad del cobre & 0.01786 \\
\hline Longitud de línea. & 15 \\
\hline Número de líneas. & 2 \\
\hline Sección del conductor & 12 AWG \\
\hline
\end{tabular}

Tabla 4 Cálculo del conductor

\section{Factor Económico (amortización)}

Tomando como ejemplo a un usuario con un consumo teórico de $850 \mathrm{kwh}$ promedio mensual, acorde a la tarifa $1 \mathrm{c}$, dicho usuario sufriría un cambio de tarifa, de 1c a tarifa DAC. Para este ejemplo se utilizó el periodo de facturación del año 2018, y en base a este se realizó un estudio económico.

En la siguiente tabla se muestra un análisis anual de facturación ya con la tarifa DAC, en donde se puede apreciar que el pago por esta tarifa es más del doble de costo que la tarifa "1C". En promedio, en la tarifa DAC se paga un coste por servicio de $\$ 4,477.63$ mientras que el promedio de pago en la tarifa " $1 \mathrm{C}$ " es de $\$ 1,926.80$. (si se incluye la instalación de un sistema fotovoltaico de $1.1 \mathrm{kw}$.) 


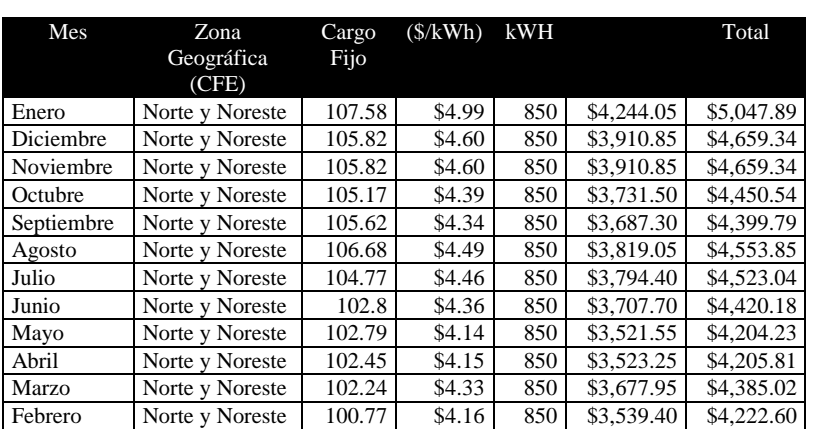

Tabla 5 Costos de tarifa DAC. (Periodo 2018)

Ahora bien, el costo por kWh en la tarifa "1C" resulta mucho más económico que la tarifa anterior, en la siguiente tabla se muestra el coste por $\mathrm{kWh}$ en las 4 formas: Consumo básico, Consumo intermedio bajo, Consumo intermedio alto y Consumo excedente.

\begin{tabular}{|c|c|c|c|c|c|c|c|}
\hline \multicolumn{2}{|c|}{ Basico } & \multicolumn{2}{|c|}{ Inter 1} & \multicolumn{2}{|c|}{ Inter 2} & \multicolumn{2}{|c|}{ EXC } \\
\hline$\$$ & 0.80 & $\$$ & 0.96 & & & $\$$ & 2.81 \\
\hline$\$$ & 0.79 & $\$$ & 0.96 & & & $\$$ & 2.80 \\
\hline$\$$ & 0.79 & $\$$ & 0.96 & & & $\$$ & 2.80 \\
\hline$\$$ & 0.79 & $\$$ & 0.96 & & & $\$$ & 2.80 \\
\hline$\$$ & 0.70 & $\$$ & 0.82 & $\$$ & 1.05 & $\$$ & 2.80 \\
\hline$\$$ & 0.70 & $\$$ & 0.82 & $\$$ & 1.05 & $\$$ & 2.80 \\
\hline$\$$ & 0.70 & $\$$ & 0.82 & $\$$ & 1.05 & $\$$ & 2.80 \\
\hline$\$$ & 0.70 & $\$$ & 0.82 & $\$$ & 1.05 & $\$$ & 2.80 \\
\hline$\$$ & 0.70 & $\$$ & 0.82 & $\$$ & 1.05 & $\$$ & 2.80 \\
\hline$\$$ & 0.70 & $\$$ & 0.82 & $\$$ & 1.05 & $\$$ & 2.80 \\
\hline$\$$ & 0.79 & $\$$ & 0.96 & & & $\$$ & 2.80 \\
\hline$\$$ & 0.79 & $\$$ & 0.96 & & & $\$$ & 2.80 \\
\hline
\end{tabular}

Tabla 6 Costo por consumo tarifa "1C" (periodo 2018)

Ahora bien, para este caso se va a implementar la generación eléctrica que proveerá de gazebo, cuyo propósito es "sacar" al usuario de la tarifa DAC, como ya se mencionó este proporcionara $1.1 \mathrm{~kW}$ para atacar este problema. La siguiente tabla muestra los datos y generación mensual.

\begin{tabular}{|c|c|l|l|l|}
\hline $\mathrm{kW}$ & \multicolumn{1}{c}{ HSP } & \multicolumn{1}{c|}{ Mes } & \multicolumn{1}{c|}{ Mes } & Generaciòn \\
\hline 1.1 & 4.38 & Enero & 31 & 149 \\
\hline 1.1 & 3.99 & Diciembre & 31 & 136 \\
\hline 1.1 & 4.45 & Noviembre & 30 & 146 \\
\hline 1.1 & 5.27 & Octubre & 31 & 179 \\
\hline 1.1 & 5.75 & Septiembre & 30 & 189 \\
\hline 1.1 & 6.37 & Agosto & 30 & 210 \\
\hline 1.1 & 6.86 & Julio & 31 & 233 \\
\hline 1.1 & 6.26 & Junio & 30 & 206 \\
\hline 1.1 & 7.27 & Mayo & 31 & 247 \\
\hline 1.1 & 6.27 & Abril & 30 & 206 \\
\hline 1.1 & 6.27 & Marzo & 31 & 213 \\
\hline 1.1 & 5.41 & Febrero & 28 & 166 \\
\hline
\end{tabular}

Tabla 7 Generación mensual (kWh) gazebo

Con la generación del gazebo frente al consumo del usuario de tarifa DAC, el resultante de consumo sería bajara y por ende el excedente.

\begin{tabular}{|r|r|r|r|r|}
\hline Consumo & Basico & \multicolumn{1}{l}{ Inter 1 } & Inter 2 & EXC \\
\hline 701 & 75 & 100 & 0 & 526 \\
\hline 714 & 75 & 100 & & 539 \\
\hline 704 & 75 & 100 & & 529 \\
\hline 671 & 75 & 100 & & 496 \\
\hline 661 & 150 & 150 & 150 & 361 \\
\hline 640 & 150 & 150 & 150 & 340 \\
\hline 617 & 150 & 150 & 150 & 317 \\
\hline 644 & 150 & 150 & 150 & 344 \\
\hline 603 & 150 & 150 & 150 & 303 \\
\hline 644 & 150 & 150 & 150 & 344 \\
\hline 637 & 75 & 100 & & 462 \\
\hline 684 & 75 & 100 & & 509 \\
\hline
\end{tabular}

Tabla 8 Consumo después de la instalación

Con base en todos los datos anteriores, se respalda el estudio económico que se expresa a continuación en las siguientes tablas, la comparación de precios de la tarifa DAC (mostrada en la tabla 5) a los precios de la tarifa " $1 \mathrm{C}$ " muestra un ahorro significativo, centrando la atención en la columna "total" de ambas tablas (5 y 9) nos damos cuenta de ello, en el mes de enero, en la tarifa DAC, el costo por consumo de energía supera los cinco mil pesos, mientras que con la tarifa " $1 \mathrm{C}$ " el costo es menor a los dos mil pesos.

\begin{tabular}{|c|c|c|c|c|c|c|c|}
\hline Basico & & Inter 1 & Inter 2 & & EXC & Total & Ahorro \\
\hline$\$ \quad 59.70$ & $\$$ & 96.00 & $\$$ & & $\$ 1,479.64$ & $\$ 1,896.99$ & $\$ 3,150.90$ \\
\hline 59.48 & $\$$ & 95.60 & $\$$ & & $\$ 1,510.28$ & $\$ 1,931.81$ & $\$ 2,727.53$ \\
\hline 59.48 & $\$$ & 95.60 & $\$$ & & $\$ 1,482.26$ & $\$ 1,899.31$ & $\$ 2,760.03$ \\
\hline 59.48 & $\$$ & 95.60 & $\$$ & & $\$ 1,389.79$ & $\$ 1,792.05$ & $\$ 2,658.49$ \\
\hline$\$ 104.55$ & $\$$ & 123.30 & $\$ 157.50$ & & $\$ 1,011.52$ & $\$ 1,620.37$ & $\$ 2,779.42$ \\
\hline$\$ 104.55$ & $\$$ & 123.30 & $\$ 157.50$ & & $\$ 952.68$ & $\$ 1,552.11$ & $\$ 3,001.73$ \\
\hline$\$ 104.55$ & $\$$ & 123.30 & $\$ 157.50$ & & 888.23 & $\$ 1,477.36$ & $\$ 3,045.68$ \\
\hline$\$ 104.55$ & $\$$ & 123.30 & $\$ 157.50$ & & 963.89 & $\$ 1,565.12$ & $\$ 2,855.06$ \\
\hline$\$ 104.55$ & $\$$ & 123.30 & $\$ 157.50$ & & 849.01 & $\$ 1,431.85$ & $\$ 2,772.38$ \\
\hline$\$ 104.55$ & $\$$ & 123.30 & $\$ 157.50$ & & 963.89 & $\$ 1,565.12$ & $\$ 2,640.70$ \\
\hline $\begin{array}{ll}\$ 59.48 \\
\end{array}$ & $\$$ & 95.60 & $\$$ & & $\$ 1,294.52$ & $\$ 1,681.53$ & $\$ 2,703.49$ \\
\hline 59.48 & $\$$ & 95.60 & $\$$ & & $\$ 1,426.22$ & $\$ 1,834.30$ & $\$ 2,388.30$ \\
\hline
\end{tabular}

Tabla 9 Costos tarifa "1C" (periodo 2018)

El ahorro del pago en tarifa DAC por 1C es significativo, en la siguiente tabla se registra el pago mensual y posteriormente el anual de la tarifa DAC.

\begin{tabular}{|l|lr|}
\hline \multicolumn{3}{|c|}{ Tarifa DAC } \\
\hline Mes & \multicolumn{2}{|c|}{ Pago mensual } \\
\hline Enero & $\$$ & $5,047.89$ \\
\hline Diciembre & $\$$ & $4,659.34$ \\
\hline Noviembre & $\$$ & $4,659.34$ \\
\hline Octubre & $\$$ & $4,450.54$ \\
\hline Septiembre & $\$$ & $4,399.79$ \\
\hline Agosto & $\$$ & $4,553.85$ \\
\hline Julio & $\$$ & $4,523.04$ \\
\hline Junio & $\$$ & $4,420.18$ \\
\hline Mayo & $\$$ & $4,204.23$ \\
\hline Abril & $\$$ & $4,205.81$ \\
\hline Marzo & $\$$ & $4,385.02$ \\
\hline Febrero & $\$$ & $4,222.60$ \\
\hline \hline Total, Anual & $\$ 53,731.62$ \\
\hline
\end{tabular}

Tabla 10 Pago DAC

Y la siguiente muestra el pago mensual y anual en la tarifa $1 \mathrm{C}$. 


\begin{tabular}{|l|lr|}
\hline Mes & \multicolumn{2}{|l|}{ Pago mensual } \\
\hline Enero & $\$$ & $1,896.99$ \\
\hline Diciembre & $\$$ & $1,931.81$ \\
\hline Noviembre & $\$$ & $1,899.31$ \\
\hline Octubre & $\$$ & $1,792.05$ \\
\hline Septiembre & $\$$ & $1,620.37$ \\
\hline Agosto & $\$$ & $1,552.11$ \\
\hline Julio & $\$$ & $1,477.36$ \\
\hline Junio & $\$$ & $1,565.12$ \\
\hline Mayo & $\$$ & $1,431.85$ \\
\hline Abril & $\$$ & $1,565.12$ \\
\hline Marzo & $\$$ & $1,681.53$ \\
\hline Febrero & $\$$ & $1,834.30$ \\
\hline Total, Anual & $\$$ & $20,247.92$ \\
\hline
\end{tabular}

Tabla 11 Pago 1C

$\mathrm{Y}$, por último, tenemos lo que registra el ahorro que implica cambar de DAC a 1C.

\begin{tabular}{|l|lr|}
\hline \multicolumn{3}{|c|}{ Ahorro total } \\
\hline Enero & $\$$ & $3,150.90$ \\
\hline Diciembre & $\$$ & $2,727.53$ \\
\hline Noviembre & $\$$ & $2,760.03$ \\
\hline Octubre & $\$$ & $2,658.49$ \\
\hline Septiembre & $\$$ & $2,779.42$ \\
\hline Agosto & $\$$ & $3,001.73$ \\
\hline Julio & $\$$ & $3,045.68$ \\
\hline Junio & $\$$ & $2,855.06$ \\
\hline Mayo & $\$$ & $2,772.38$ \\
\hline Abril & $\$$ & $2,640.70$ \\
\hline Marzo & $\$$ & $2,703.49$ \\
\hline Febrero & $\$$ & $2,388.30$ \\
\hline Ahorro anual & $\$$ & $33,483.70$ \\
\hline
\end{tabular}

Tabla 12 Ahorro con Gazebo

Como se aprecia en la anterior, el cliente se ahorraría más de treinta mil pesos al año. El costo de la inversión al instalar el gazebo es de $\pm \$ 47,000.00$ y el tiempo de retorno de la inversión hacia el cliente es de 1.5 años, que está dentro del periodo establecido por FIDE, de acuerdo con la revista "Eficiencia energética" y cito: "Financiamientos atractivos. Plazo de los créditos hasta por cinco años, con periodo simple de recuperación de hasta siete años para usuarios con tarifa 2 , y de hasta 10 años para usuarios con tarifa 3, OM y HM" (elèctrica., 2018)

\section{Diseño Preliminar}

La siguiente figura muestra la parte superior del gazebo, en donde la parte sombreada con color café representa el techo y la parte de color gris representa los paneles. Este diseño preliminar fue elaborado en el software de diseño mecánico Inventor $®$.

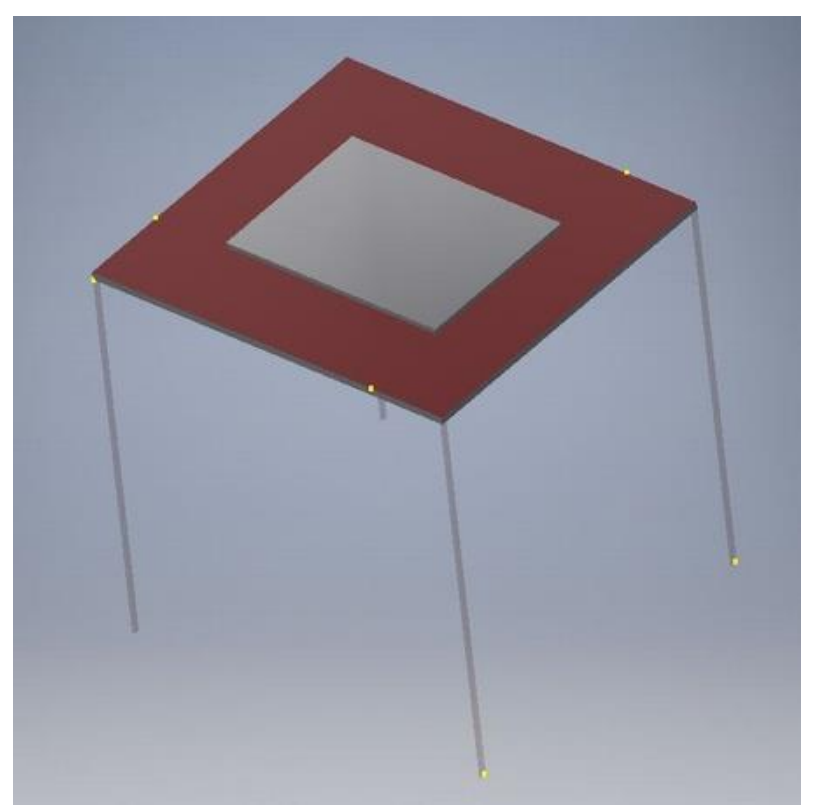

Figura 5 Vista superior del boceto

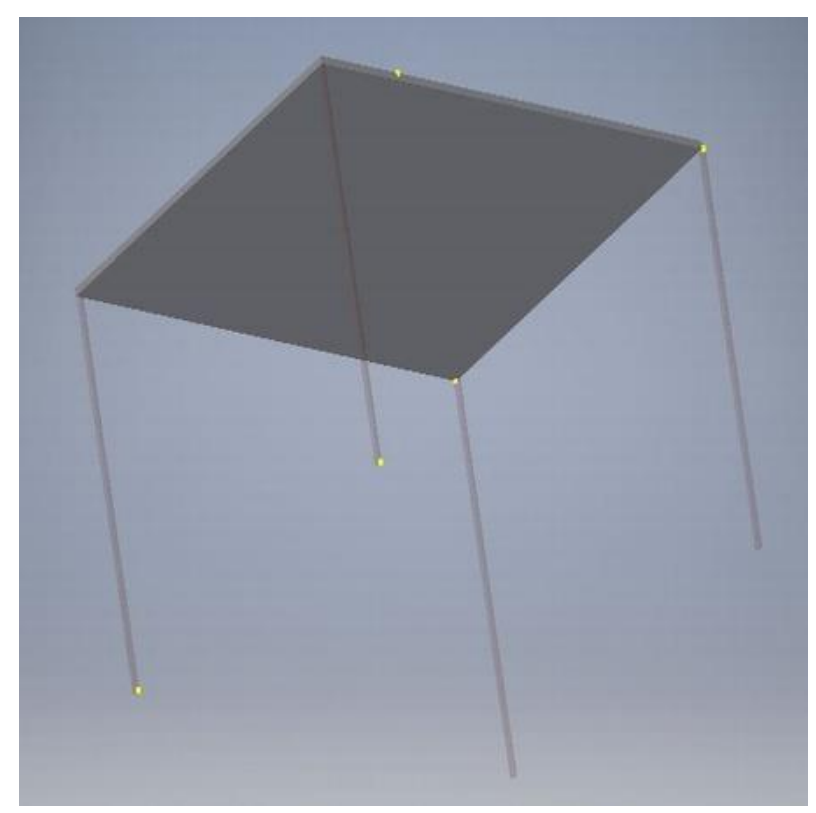

Figura 6 Vista inferior del boceto

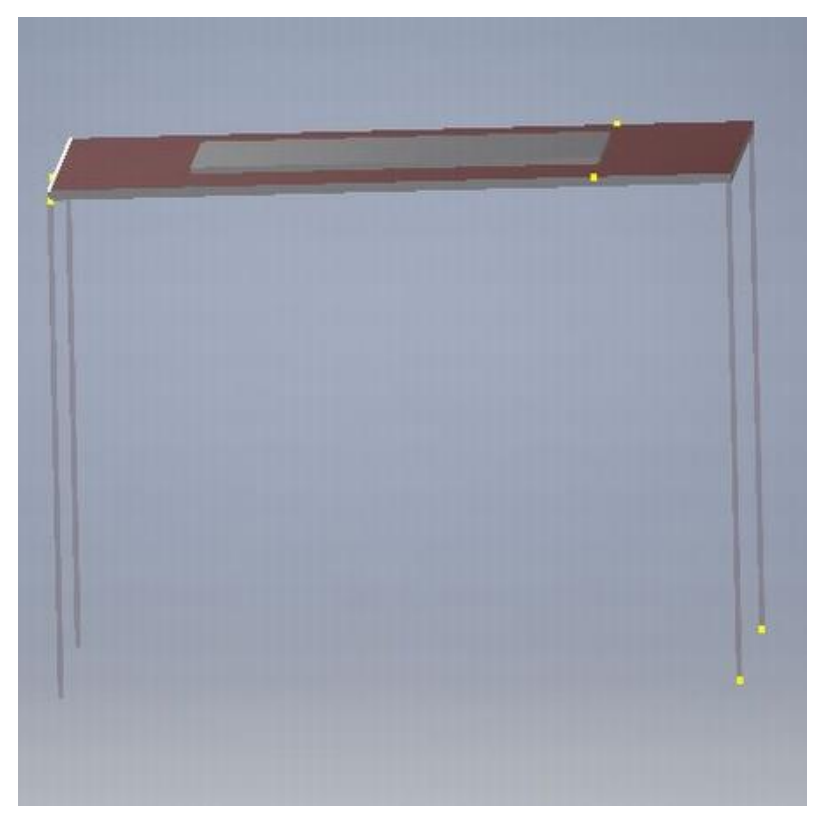

Figura 7 Vista lateral del boceto

SÁNCHEZ-CORTEZ, José Alfonso, CASTILLO-RAMÍREZ, Carlos Eduardo, VÁZQUEZ-BALDAZO, Luis Guillermo y GONZALEZMORALES, Amparo. Diseño de un sistema fotovoltaico para la reducción de usuarios de tarifa DAC. Revista de Innovación Sistemática. 2019 


\section{Resultados}

Actualmente se cuenta con 3 usuarios con tarifa DAC, se están realizando diagnósticos energéticos y evaluaciones para determinar si necesita un prototipo del "gazebo" o mejorar su condición energética (chequeo de instalación eléctrica, uso que le da a la energía, aparatos obsoletos etc.) De ser elegibles para un gazebo se comenzar con los tramites pertinentes que conlleva un sistema interconectado.

El desarrollo del proyecto ha aportado nuevos conocimientos a todos los involucrados, ingenieros, alumnos, docentes auxiliares etc. La proyección a futuro es comercializar un catálogo de gazebos personalizados y optimizados según la necesidad del cliente.

\section{Agradecimientos}

Agradecimientos a la Rectoría de la Universidad, a cargo del Ing. Isaac Rebaj Sevcovicius.

A la dirección de la carrera de Energías Renovables, quienes han facilitado todo lo necesario y han hecho posible el desarrollo del proyecto, reconocimientos especiales a las siguientes personas:

Director de la carrera de mecatrónica y energías renovables: Ing. Juan H. Almazán Covarrubias. Por el apoyo prestado, las instalaciones, material eléctrico y fotovoltaico, así como los equipos.

Al ingeniero: M.C José Alfonso Sánchez Cortez. Principal responsable del proyecto, quien ha gestionado todos y cada uno de los puntos a desarrollar y quien también ha colaborado con la redacción es este artículo.

Dra. Amparo González Morales. Por ser la responsable de la supervisión y edición de este artículo, así como su directa colaboración en el proyecto.

T.S.U Eduardo Castillo Ramirez y T.S.U Luis Guillermo Vázquez Baldazo por su apoyo y directa colaboración.

\section{Conclusiones}

Seguir trabajando en este proyecto, aprender sobre "la marcha" todo lo necesario y sobre todo "pulir" detalles en el mismo para que así, este muy próxima su comercialización.

Ayudar a todos los usuarios posibles a salir de esta tarifa, ayudarlos en su economía y concientizarlos sobre una correcta educación energética.

\section{Referencias}

(SENER), S. d. (2019). Inventario Nacional de Energías Limpias. Obtenido de INEL: https://dgel.energia.gob.mx/inel/mapa.html?lan $\mathrm{g}=\mathrm{es}$

(2019 de Agosto de 29). Obtenido de Comisión Federal de Electricidad.

Comisión Feder. (28 de Agosto de 2019). Obtenido de https://app.cfe.mx/Aplicaciones/CCFE/Tarifas/ TarifasCRECasa/Tarifas/TarifaDAC.aspx Comision Reguladora de Energía. (2017).

Cortez, J. A. (2019). Parasol Solar. Altamira.

eléctrica., F. p. (2011). Financiamiento para la Generación de Energía Eléctrica con Sistemas Fotovoltaicos. 3.

elèctrica., F. p. (2018). Eficiencia Energetica. INCORPORACIÓN DE SISTEMAS FOTOVOLTAICOS AL PROGRAMA ECOCRÉDITO EMPRESARIAL, 6-8.

México., G. d. (19 de 06 de 2018). Gobierno de México. Obtenido de Gobierno de México.: https://datos.gob.mx/busca/dataset/usuarios-yconsumo-de-electricidad-por-municipio-apartir-de-2018

SENER. (2018). Balance Nacional de Energia. México.

SENER. (2018). Balance Nacional de Energía 2017. México: Secretaria de Energía. 